\title{
1 Reverse Correlation and the VESPA Method
}

\author{
Edmund C. Lalor, Barak A. Pearlmutter, and John J. Foxe
}

The traditional method of obtaining event-related potentials (ERPs) typically involves the repeated presentation of discrete stimulus events and extraction from the ongoing neural activity using signal averaging techniques. Assuming a sufficiently high sampling rate, this technique allows for the determination of a response whose individual components are clearly resolved in time, allowing for a temporally detailed analysis of sensory and cognitive processes. While this method has led to tremendous advances in our understanding of the brain, both healthy and otherwise, it has a number of intrinsic limitations. These include the inability to adequately resolve responses to more than one stimulus at a time, the nonenvironmental and somewhat aversive nature of suddenly onsetting stimuli, particularly in the visual domain, and the lengthy acquisition time resulting from the incorporation of a sufficient delay between stimuli to allow the neural activity to return to baseline.

In this chapter we describe a method for obtaining a novel visual ERP known as the VESPA (for visual evoked spread spectrum analysis) that seeks to address the limitations of the standard visual evoked potential (VEP). This method involves the recording of neural activity during the presentation of continuous, stochastically modulated stimuli and the use of reverse correlation to determine the transfer function of the human visual system, that is, the function that converts the presented stimulus into the recorded neural activity. First, we introduce the broader reverse correlation technique that has seen widespread use in the analysis of many physiological systems. We follow this introduction with a description of the VESPA method itself, including a discussion of the differences between the standard VEP and the VESPA, and some proposed applications and extensions.

\section{Analysis of Physiological Systems Using Stochastic Stimuli}

In order to gain some useful understanding of complex physiological systems, especially considering the inherent difficulty of accessing the inner details of such systems as they function, one must first make some simplifying assumptions. This allows one 
to infer models of the system based on observations of its behavior under certain conditions. One approach that has met with some success is the application of system identification methodologies to the analysis of physiological systems. That is to say, one can attempt to obtain a mathematical expression for the system functional, $S(\bullet)$, that converts a given input, $x$, into a measured output, $y$, even in the presence of measured noise, $w$, and unmeasured noise $z$ (see figure 1.1). This is usually accomplished in two steps. First, given all prior knowledge of the system, an appropriate model is chosen. Then, in the second step, the parameters of this model are estimated based on the output of the system corresponding to particular inputs.

The most widely studied and well understood class of dynamical systems considered in practice and in the system identification literature are so-called linear time-invariant (LTI) systems. Study of these systems involves the assumption that the output depends linearly on the input and entails estimation of the impulse response of the system. Despite the obviously unrealistic assumption about the real life processes they represent, the approximations employed are often reasonable and the linear models obtained often lead to useful results. However, a considerable amount of research has also been done extending this methodology to the modeling of nonlinear time-invariant systems. Most of this work is based on the mathematics of the VolterraWiener approach. The Volterra series was first studied by Vito Volterra around 1880 as a generalization of the Taylor series of a function. Norbert Wiener (1958) used the Volterra series to model the input-output relationship of a nonlinear system. This technique allows one to define a set of discrete Volterra kernels that completely characterize the nonlinear system under investigation. It does so by using a Gaussian time function as an input that allows for the estimation of a set of Wiener kernels that can then be used to determine the model's Volterra kernels. Lee and Schetzen (1965) described a practical method for determining the Wiener kernels by cross-correlating the system response with its white Gaussian input. Schetzen (1981) provides a good review of nonlinear system modeling based on the Wiener theory.

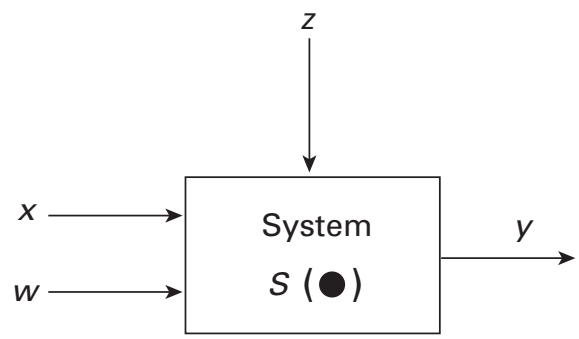

Figure 1.1

System with output $y$, input $x$, measured noise $w$, unmeasured noise $z$, and system functional $S(\bullet)$. 
The primary application of this type of nonlinear system modeling based on the Wiener theory is in the experimental identification of physiological systems (Hung and Stark 1977; Marmarelis and Marmarelis 1978; Marmarelis 2004). As mentioned, linear assumptions can often lead to useful information about a system. However, it is clear that physiological systems are highly nonlinear and that their function is often critically dependent on those nonlinearities. One important consideration in physiological modeling is the type of input that is used to estimate the model of the system. The white noise approach (deBoer and Kuyper 1968; Marmarelis and Marmarelis 1978) proposes utilizing widely varying input-output data in order to span the full dynamic operating range of the system rather than simplified, less natural data that may ignore large parts of this range. By employing spectrally and temporally rich input signals such as white noise to explore the behavior of the system, one maximizes the amount of power being used to drive and thus interrogate the system under investigation.

Several successful examples of the use of nonlinear modeling have been reported across different physiological domains (Marmarelis and Naka 1972; for review, see Marmarelis 2004). In particular, a large number of studies of the visual system have utilized quasiwhite light stimulation in order to derive a model of the behavior of cells, from photoreceptors through to simple V1 cells (Marmarelis and McCann 1977; Sakai and Naka 1987, Meister et al. 1994; Jones and Palmer 1987; DeAngelis et al. 1993). At a more macroscopic scale, the Volterra-Wiener approach has been applied to EEG and, in particular, the visual evoked potential (Coppola 1979). In recent work (Lalor et al. 2006) we have revisited this idea and developed a method for evoking a novel ERP known as the VESPA that seeks to harness the technique of reverse correlation and, in so doing, address various limitations of the traditional VEP approach.

\section{The VESPA Method}

Following the white noise approach, and unlike the traditional VEP technique, the VESPA is elicited using continuous, stochastic input to the visual system. This is accomplished by rapidly modulating either the contrast or mean luminance of a visual stimulus using a precomputed signal with Gaussian distribution. For example, one can generate a number of checkerboard images of different contrasts and then present a different one on every refresh of a computer monitor as determined by the precomputed signal. Because there is not much EEG or MEG power above $30 \mathrm{~Hz}$, a refresh rate of $60 \mathrm{~Hz}$ seems sensible and works well. The modulation signals used to elicit the VESPAs shown in the present chapter had their power spread uniformly over the 0 to $30 \mathrm{~Hz}$ range. The fundamental difference between the VEP and VESPA stimulation is illustrated in figure 1.2. 

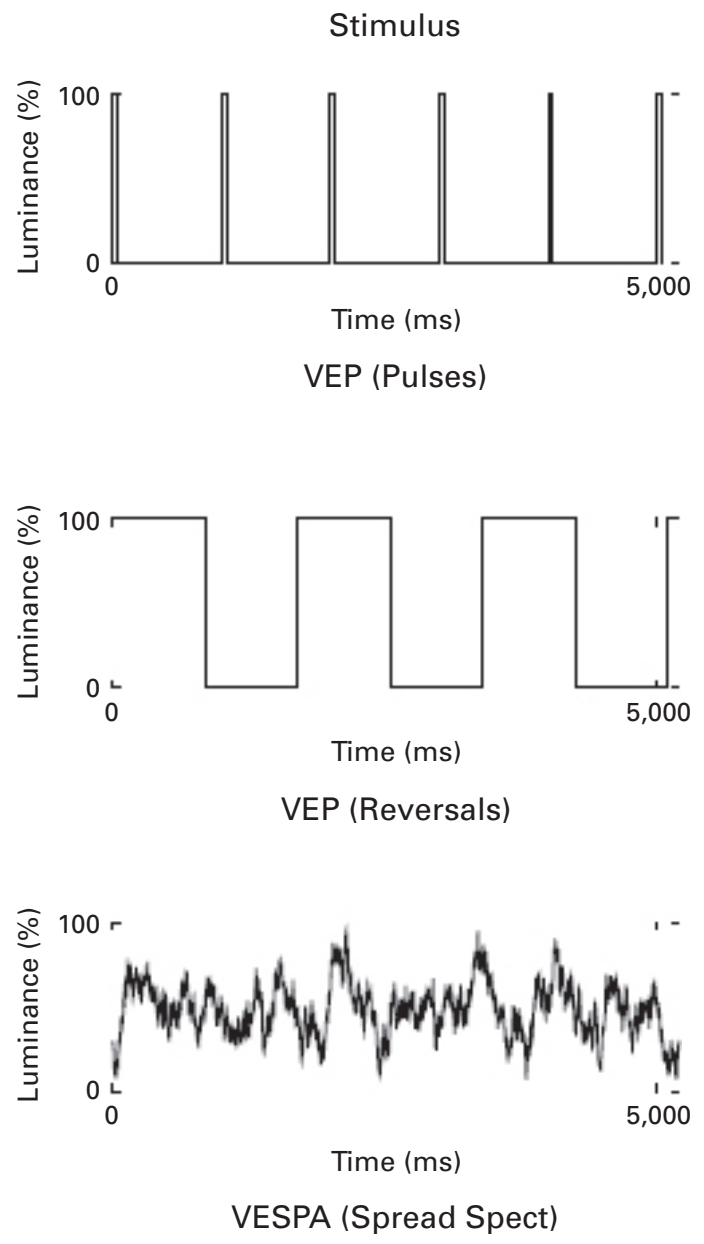

Figure 1.2

Basic difference between VEP and VESPA stimuli. Pulsed and pattern-reversal VEP stimuli modulated from 0 to 100 percent in discrete jumps isolated in time. The VESPA stimulus smoothly but stochastically modulates across the range 0 to 100 percent with many intermediate values. A Gaussian distribution for this modulating signal works well. 
As shown in figure 1.3, the VESPA model assumes that the EEG or MEG response, $y(t)$, consists of a convolution of this stochastic input signal, $x(t)$, with an unknown impulse response waveform $w(\tau)$, plus noise,

$y(t)=x(t) \bullet w(\tau)+$ noise.

Given the precomputed stimulus waveform, $x(t)$, and the measured EEG or MEG signals, $y(t)$, we can estimate the values of the impulse response function, $w(\tau)$, using linear least squares estimation (for details, see appendix A). The impulse response $w(\tau)$ is known as the VESPA. The EEG or MEG can be thought of as the superposition of many impulse responses (i.e., VESPAs), one per frame, each scaled by the associated input value (see figure 1.3).

The VESPA is plotted in $\mu \mathrm{V}$ on a time axis that indicates the relationship between the (normalized; see appendix A) incoming stimulus signal and the output EEG a certain time later. For example, the $0 \mathrm{~ms}$ time point of a VESPA waveform indicates the relationship between the input stimulus and the EEG at exactly the same time, which should be zero given that the response to a stimulus does not propagate through the visual system in 0 ms! Typically the VESPA is nonzero in an interval from around 50 to $250 \mathrm{~ms}$ indicating that each input stimulus affects the EEG across a range of time 50 to $250 \mathrm{~ms}$ later, which was already known from use of the standard VEP.

\section{Comparison to VEP}

The similar morphologies of the VESPA and VEP are clearly seen in figure 1.4. While these responses show a high degree of correlation $\left(r=0.91, p<10^{-28}\right)$, the within subject correlations between responses that make up the group averages are typically much lower (Lalor et al. 2006). This suggests that the VESPA and VEP give largely similar information across a group, but that they may give complementary information on an individual basis.

In fact it can be argued that the VESPA method is a strict generalization of the VEP technique. In the special case of the VEP the stimulus, $x(t)$, consists of a set of widely spaced impulses which, when processed using the VESPA analysis described herein, leads to a straightforward averaging procedure. The more general VESPA analysis allows for the incorporation of a whole range of stimulus classes such as on/off discontinuities, periodic bursts, and changes in temporal frequency, and thus for a much more thorough investigation of the properties of the visual system than discrete stimulation alone.

Another advantage of the VESPA is illustrated in figure 1.5, which shows a plot comparing the signal-to-noise ratios (SNRs) achieved by the VESPA and VEP using the same basic checkerboard stimulus at electrode location Oz. The SNR was calculated at $5,000 \mathrm{~ms}$ intervals by defining the noise as the mean of the squared values in the 


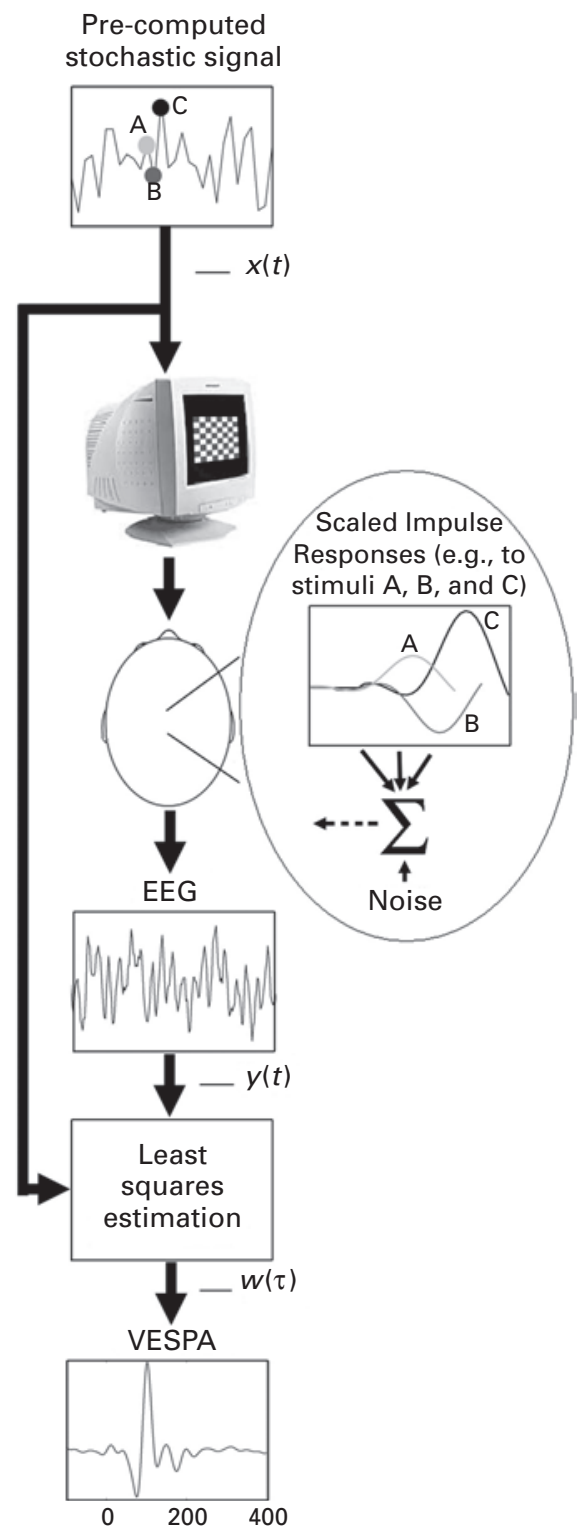

Figure 1.3

Flow diagram of VESPA acquisition. The EEG or MEG is modeled as a sum of overlapping impulse responses scaled by the corresponding stimuli, plus noise. Three such scaled impulse responses are shown, corresponding to stimulus values $\mathrm{A}, \mathrm{B}$, and $\mathrm{C}$. 


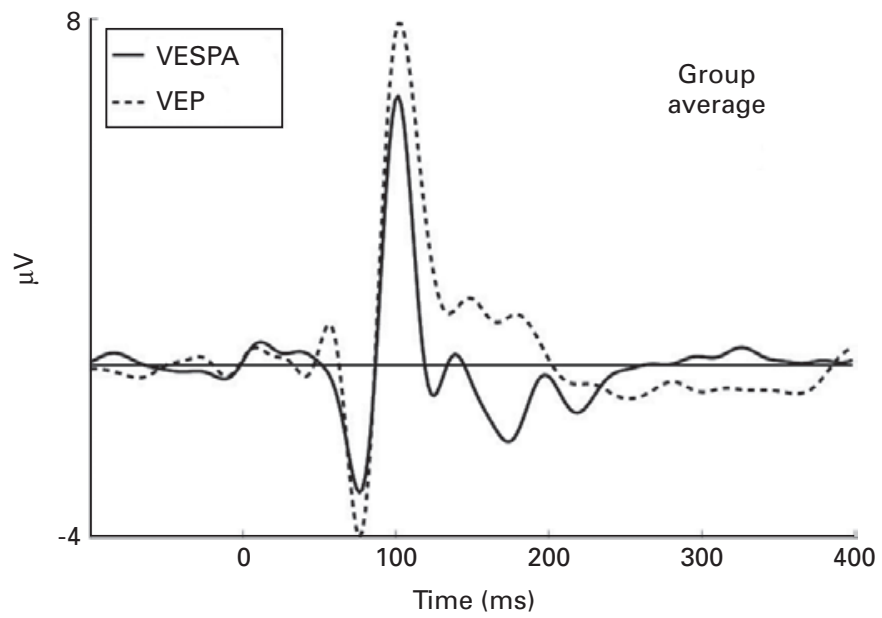

Figure 1.4

VESPA elicited by stochastic modulation of the contrast of a checkerboard stimulus and the standard VEP obtained in response to the same checkerboard pattern reversing in phase every second. Both responses are for data at electrode location $\mathrm{Oz}$ and are averaged across a group of 10 subjects, each of whom underwent two 120 s runs for each method.

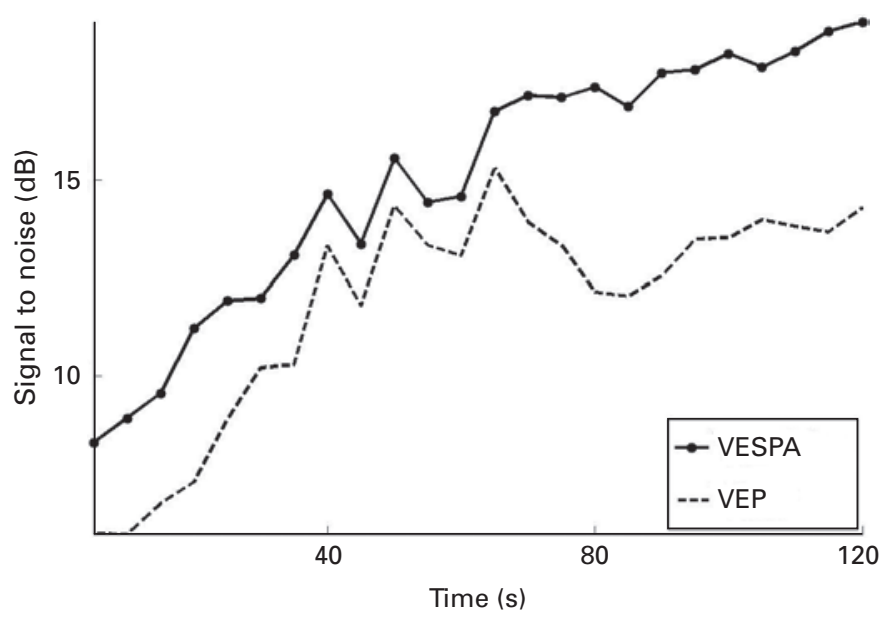

Figure 1.5

Signal-to-noise ratio as a function of acquisition time for the VESPA and VEP elicited by checkerboard stimuli at electrode location Oz. SNRs were calculated for VESPAs and VEPs averaged across two 120 s runs for 12 subjects. 
$100 \mathrm{~ms}$ interval immediately preceding the stimulus and the signal as the mean of the squared values in the interval 35 to 175 ms poststimulus. The VESPA achieves a SNR higher than that obtained using the standard method at every time point and is almost $5 \mathrm{~dB}$ higher after $120 \mathrm{~s}$.

\section{Extension to Quadratic Terms}

The standard linear VESPA method described above can easily be extended to higher orders. For example, in the case of a quadratic analysis, this is accomplished by including in the least squares estimation not only the first-order values of the modulating signal within the desired window but also all second-order products of these values (for details, see appendix B or Lalor et al. 2008). This allows us to determine how the EEG depends not only on the individual input signal values but also on interactions between inputs at different time lags. For example, the VESPA value at $(75,100)$ ms indicates the relationship between the EEG or MEG and the interaction between the input stimulus values 75 and $100 \mathrm{~ms}$ earlier. Figure 1.6 illustrates the average quadratic VESPA response at electrode location $\mathrm{Oz}$ for data gathered from 11 subjects each of whom undertook 10 minutes of testing. To reduce processing time, the estimation was restricted to a $120 \mathrm{~ms}$ window starting $20 \mathrm{~ms}$ poststimulus. A strong positive

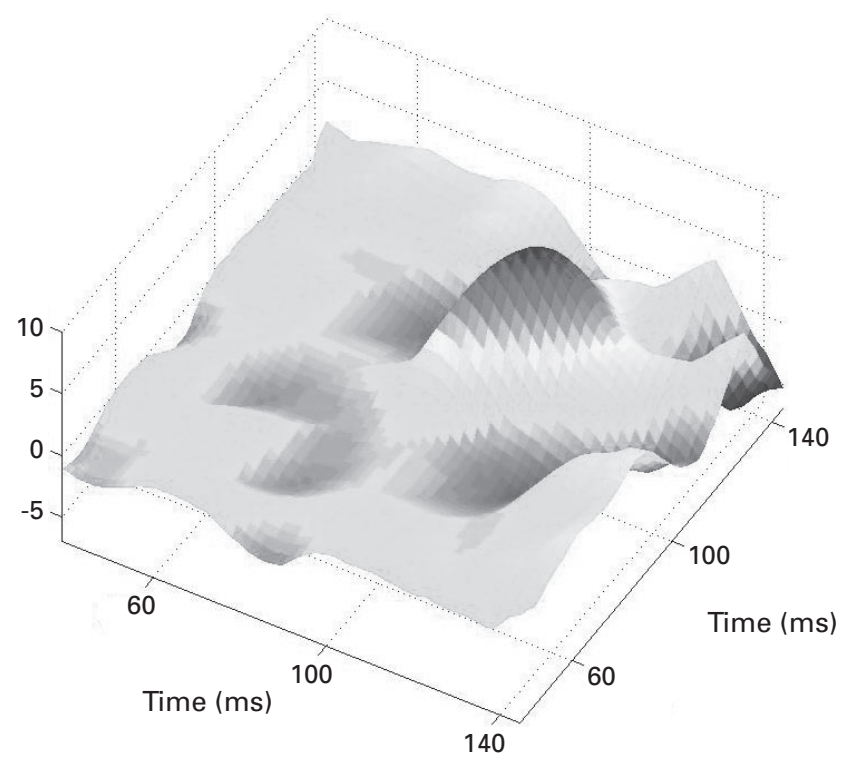

Figure 1.6

Surface plot of the second-order VESPA response at electrode location Oz for data gathered from 11 subjects, each of whom undertook 10 minutes of testing. 
relationship can be seen between the EEG and the input stimulus interactions around $(100,100) \mathrm{ms}$, which is analogous to the positive component around $100 \mathrm{~ms}$ in the case of the linear VESPA. Some nonzero VESPA values can be seen off the diagonal, indicating that the second-order VESPA carries information not present in the linear VESPA. Further work will elucidate the relationship between the relative information contained in the linear and the nonlinear VESPA.

\section{Cell Populations Activated by the VESPA and the VEP}

The fact that the stimuli used to elicit the VESPA and VEP are so different begs the question: Do they represent activity of the same neuronal populations? Figure 1.7 shows a scalp topography for the VESPA (bottom row) that is quite distinct from that of the VEP (top row). The abiding characteristic of the early VESPA maps is the persistently delimited focus over midline occipital scalp without any evidence for the characteristic early bilateral spread over lateral occipital scalp regions that is consistently seen for the standard VEP (Gomez-Gonzalez et al. 1994; Foxe and Simpson 2002). This pattern suggests that the VESPA may well have a distinct cellular activation pattern from that of the VEP, favoring midline structures such as striate cortex and neighboring retinotopically mapped extrastriate regions as well as regions in the dorsal visual stream, activation of which is known to produce midline scalp topographies. Previous

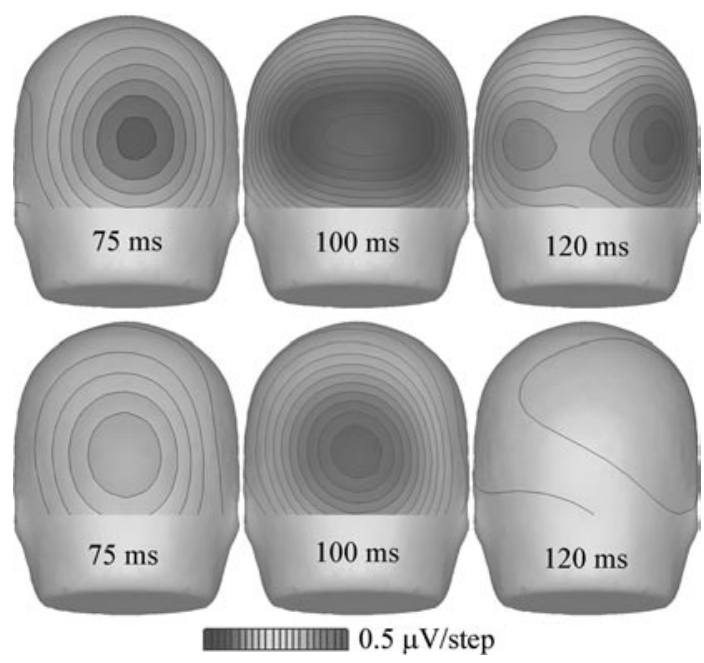

Figure 1.7

Topographic maps indicating the scalp distribution of the VEP (top row) and the VESPA (bottom row) at 75, 100, and $120 \mathrm{~ms}$. Activity at $100 \mathrm{~ms}$ is much more focused over the midline for the VESPA. The bilateral spread evident at $120 \mathrm{~ms}$ for the VEP is not manifest in the VESPA. (See plate I.) 
studies have shown that the bilateral maps found during the early components of the VEP represent, in large part, activation of structures in the ventral visual stream such as the lateral occipital complex (Doniger et al. 2001; Foxe and Simpson 2002; Murray et al. 2004). Therefore one implication of this major difference is that the parvocellular system, which provides the major input to the ventral stream, may not be effectively activated by the VESPA.

This hypothesis can be considered with regards to the stimulus preferences of magno and parvo cells. Parvo cells with their spectrally opponent nature are know to be less sensitive to luminance contrast than magno cells (Kaplan et al. 1990; Lee et al. 1990). While the high contrast gain of cells in the magnocellular pathway might suggest that they may be more sensitive to the contrast modulations of the VESPA stimulus, their response saturates at fairly low contrasts (10-15 percent; e.g., Baseler and Sutter 1997). Parvocellular neurons meanwhile have lower contrast gain but do not saturate (see Butler et al. 2007). Furthermore it has been suggested that the temporal responses of the parvocellular system are much more linear than those of magnocellular cells (Kaplan and Benardete 2001). Given that the stimulus used to generate the VESPA shown in figure 1.7 spent less than 2 percent of its time below 15 percent contrast (Lalor et al. 2006), and that the analysis method used was strictly linear, it seems reasonable to conclude that this VESPA may actually reflect mostly activity of parvocellular pathways.

How then to explain the dramatic differences in scalp distribution and in particular the fact that, unlike the VEP, there is no lateralization of the P1 component for the VESPA? One good reason is that the VESPA analysis used assumes that the measured EEG is linearly related to the modulation of the simple luminance or contrast feature of the input stimulus. It is likely that this assumption holds truest for the relatively simple cells of early visual areas, particularly those in striate cortex, and less strongly for complex cells in higher order areas such as in parietal or inferotemporal (IT) cortex. Furthermore regions like IT are optimized for object recognition and thus are presumably not well suited to the analysis of stationary objects flickering at $60 \mathrm{~Hz}$.

In order to further investigate the cellular origins of the VESPA, one can exploit the flexibility of the method to alter the characteristics of the stimulus. For example, one could adjust the temporal frequency statistics and/or the range over which the stimulus modulates in order to bias one subsystem over the other. Figure 1.8 shows VESPA responses to stimuli biased toward magnocellular and parvocellular pathways through restriction of the contrast modulation to within ranges of 0 to 10 percent and 32 to 100 percent respectively.

The dramatically different morphologies of these VESPA response strongly suggest that they index activity of distinct subpopulations of cells and is an example of the flexibility of the VESPA method. It is also clear from this figure that the parvocellularly biased VESPA much more closely resembles the standard VESPA, providing further 


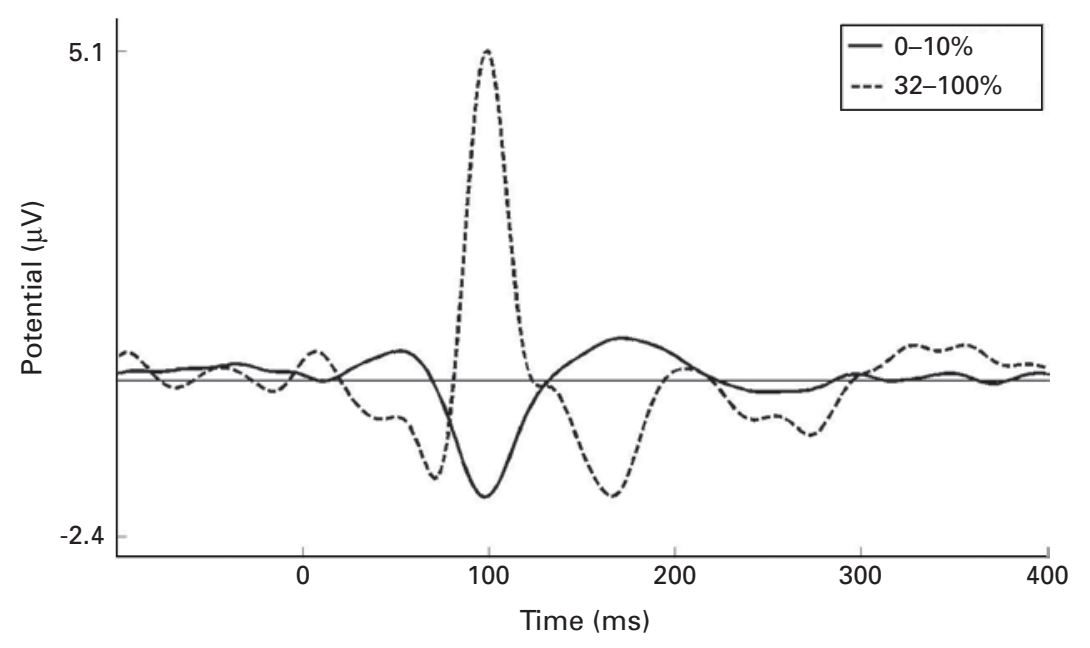

Figure 1.8

Plot of VESPA responses to stimuli whose contrast modulation was restricted to 0 to 10 percent and 32 to 100 percent. The 0 to 10 percent stimulus likely preferentially stimulates the magnocellular pathway, with the 32 to 100 percent stimulus reflecting activity mostly from parvo cells.

evidence that the standard VESPA indexes activity of parvo cells. (See Lalor and Foxe 2008 for more details.)

The ability to obtain responses biased toward one subsystem over the other could be very useful in both research and clinical settings. Aside from studies designed to gain a better insight into the processing mechanisms of the magnocellular and parvocellular pathways in general, each of these subsystems has also been studied in patients with various eye disorders such as retinitis pigmentosa (Alexander et al. 2004, 2005), glaucoma (McKendrick et al. 2007) and strabismic amblyopia (Davis et al. 2006). Furthermore magnocellular pathway function has been reported to be relatively more impaired in neurocognitive disorders such as schizophrenia (Foxe et al. 2005; Butler et al. 2005, 2007; Kim et al. 2005) and dyslexia (Chase and Stein 2003). Studies utilizing the standard VEP have moreover consistently demonstrated that patients with schizophrenia exhibit relatively severe deficits in early visual sensory processing, as indexed by a robust decrement in amplitude of the occipital P1 component (e.g., Foxe et al. 2001, 2005; Butler et al. 2001, 2007; Doniger et al. 2002; Spencer et al. 2003; Schechter et al. 2005; Haenschel et al. 2007). In a first investigation of the VESPA in schizophrenia, the standard VESPA from a group of patients was shown to be virtually identical to that from a group of controls, with the same two groups displaying dramatic differences in the P1 component of their respective VEPs (Lalor et al. 2008). 
This finding clearly shows that the information provided by the VESPA and VEP is not identical and that the VESPA may be of great use in clinical investigation, perhaps in conjunction with the standard VEP. A second study involving patients with schizophrenia is underway using the magnocellularly biased VESPA.

\section{Multiple Simultaneous Stimuli}

One of the major advantages of the VESPA method is the ability to obtain separate responses to multiple simultaneously presented stimuli. This is accomplished by placing stimuli in distinct regions of the visual field and modulating those stimuli using mutually orthogonal stochastic signals. It is not necessary that the modulating signals have different frequency content. They can in fact have exactly the same statistics; that is, they can simply be different instantiations of the same random process. Two VESPAs acquired from data at electrode location $\mathrm{Oz}$ in response to two concurrently presented bilateral stimuli are shown in the left panel of figure 1.9. The responses shown represent the average across a group of ten subjects, each of whom underwent two 120 s runs.

An obvious application of this ability is to the study of visual spatial attention. Visual attention is largely controlled endogenously but is also affected exogenously through the influence of novel stimuli and events in our visual field. While ERP/VEP studies investigating endogenous attention have been widely reported, these experiments have a serious limitation in that the suddenly onsetting stimuli used to elicit the ERP inevitably have an exogenous, attention-grabbing effect on the subject. Using the VESPA method, it is possible to investigate the effect of endogenous spatial attention on visual processing with excellent temporal resolution and without the use of suddenly onsetting stimuli. Furthermore, unlike with the traditional VEP, it is possible to emulate the more common real-life situation where both relevant and irrelevant information are present in the visual field at the same time. Obtaining VESPAs to attended and unattended stimuli simultaneously affords greater flexibility in the investigation of the mechanisms of enhancement and suppression in attentional deployment. The effect of attention on the VESPA in response to one of two simultaneously presented stimuli is shown in the right panel of figure 1.9. See Lalor et al. (2007) for more details of the use of the VESPA for the study of visual spatial attention.

\section{Extensions}

The approach outlined for the VESPA in this chapter could also be used to investigate other sensory systems. For example, an auditory analog of the VESPA could be elicited by stochastically modulating the amplitude of an auditory carrier signal. As with the VESPA, this method could afford a great deal of flexibility in the design of complex stimuli, allowing for a thorough analysis of the properties of the sensory system under 


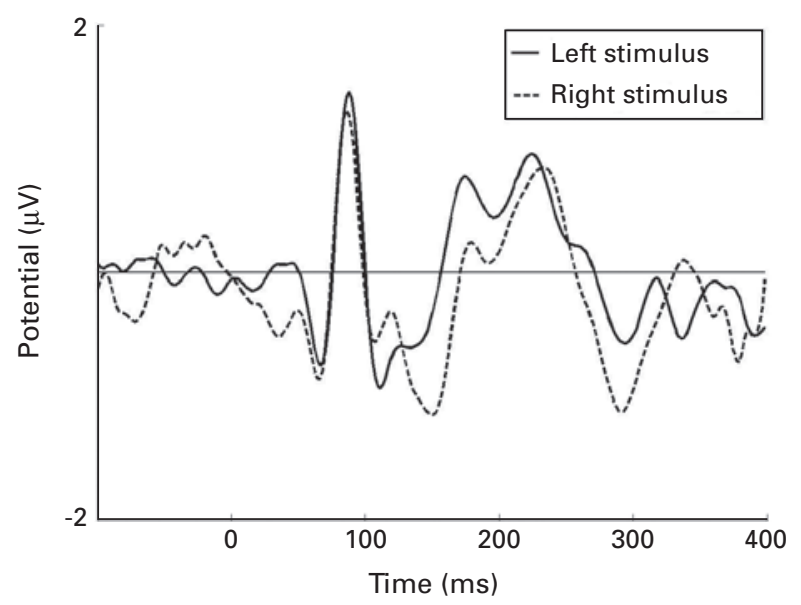

a

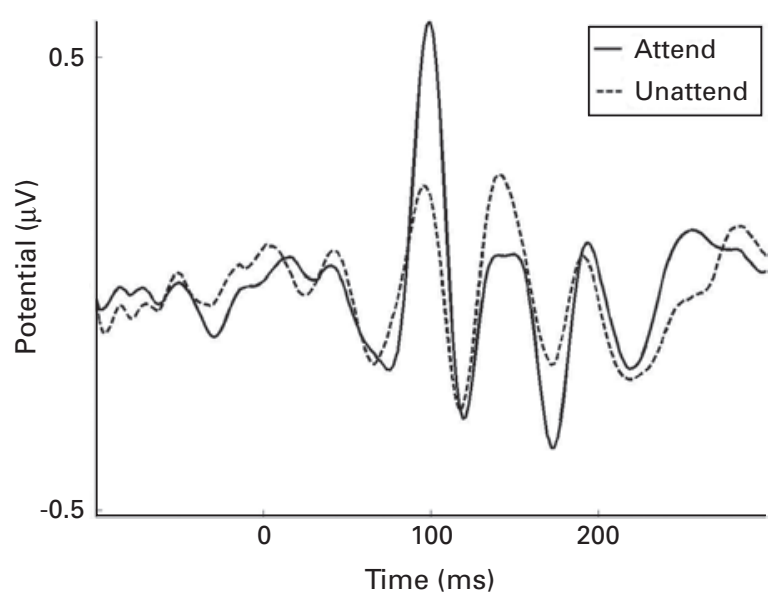

b

Figure 1.9

(a) Two VESPA responses obtained to concurrent, bilaterally presented stimuli. The responses shown represent the average across a group of 10 subjects, each of whom underwent two $120 \mathrm{~s}$ runs and are from data at electrode location Oz. (b) The effect of visual spatial attention on one of two simultaneously presented VESPA stimuli. Notice the significant enhancement of the peak around $100 \mathrm{~ms}$ in the attended condition. This plot represents the average response at $\mathrm{Oz}$ from a group of 15 subjects, each of whom underwent 50 trials of $40 \mathrm{~s}$ for both attend and unattend conditions. 


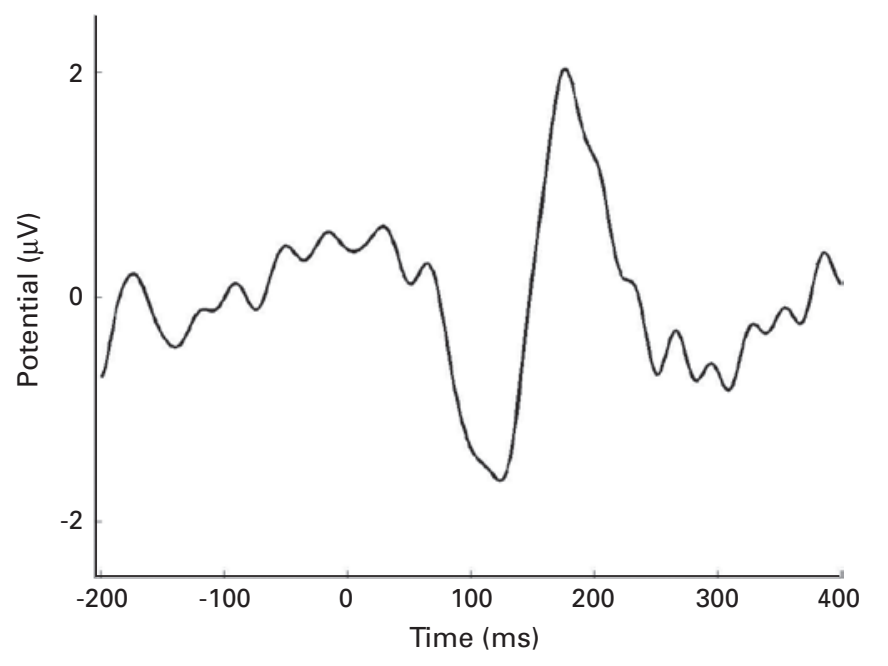

Figure 1.10

Auditory analogue to the VESPA response at electrode location Fz (referenced to the left mastoid) to a $1 \mathrm{kHz}$ audio signal whose intensity was modulated by a stochastic signal with power spread uniformly over the range 0 to $30 \mathrm{~Hz}$. The response shown was obtained from one subject who undertook ten $120 \mathrm{~s}$ runs.

investigation. A plot of an auditory analog to the VESPA obtained from one subject using an amplitude modulated $1 \mathrm{kHz}$ auditory tone can be seen in figure 1.10.

The auditory and visual responses shown in this chapter also suggest the utility of the VESPA method in investigation of multisensory integration processes (Foxe and Schroeder 2005). By mutual manipulation of the statistics of the auditory and visual modulation signals one could determine the sensitivity of multisensory processing as a function of the correlation between the temporal variations in the different modalities.

\section{Summary}

We have described a method for obtaining a novel robust visual evoked response, known as the VESPA, that can be rapidly and continuously obtained using one or more simultaneously presented, unobtrusive stimuli. It is hoped that the VESPA method will prove to be a useful tool for research into schizophrenia and other areas as well as in clinical practice. The ease with which multiple spread spectrum stimuli can be incorporated into more natural experimental paradigms should render them very useful in studies which require short time monitoring of endogenous visual 
attention as well as in the design of brain-computer interfaces. Extensions to research on other modalities have been shown, and further extensions proposed.

\section{Appendix A: Estimation of the VESPA}

To perform the least squares fit we form the $n$-dimensional column vector $x_{t}$ consisting of the sampled points of the precomputed modulating input signal

$\left(x\left(t-t_{0}\right), x\left(t-\left(t_{0}+1\right)\right), \ldots, x\left(t-\left(t_{0}+n-1\right)\right)\right)^{T}$,

where $n$ is the number of sampled points of the impulse response function, $w$, that we wish to estimate and $t_{0}$ is the estimation window offset. The values for $t_{0}$ and $n$ used in this chapter, for example, were $-100 \mathrm{~ms}$ and $500 \mathrm{~ms}$ respectively. The values of $x_{t}$ are normalized to between 0 and 1 and are then scaled by multiplying by the refresh rate of the monitor and dividing by the sampling rate of the acquisition device. This is to take into account the fact that the input stimulus and the data are both discrete in time and that the sampling rate of each affects the estimated values of the impulse response in a linear fashion. We can then estimate the $n$-dimensional vector, $w$, consisting of the sampled points of the response function

$\left(w\left(t_{0}\right), w\left(t_{0}+1\right), \ldots, w\left(t_{0}+n-1\right)\right)^{T}$

by minimizing

$E=\left\langle\left\|w^{T} x_{t}-y_{t}\right\|^{2}\right\rangle=w^{T}\left\langle x_{t} x_{t}^{T}\right\rangle w-2 w^{T}\left\langle x_{t} y_{t}\right\rangle+\left\langle y_{t} y_{t}\right\rangle$,

where $\langle\bullet\rangle$ indicates an average over $t$.

Expanding $d E / d w=0$ gives

$w=\left\langle x_{t} x_{t}^{T}\right\rangle^{-1}\left\langle x_{t} y_{t}\right\rangle$.

Here $w$ can be solved for straightforwardly by first constructing the $n \times n$ matrix $x_{t} x_{t}^{T}$ at each time point of our stimulus signal, determining a running sum across all time points and dividing the sum by the number of time points. Second, the $n$-dimensional vector $x_{t} y_{t}$ is calculated, again at each time point and the mean is determined across all time points, again using a running sum. The final step involves a simple matrix inversion and multiplication.

We can further improve the quality of our estimate by adding a regularization term. This serves to increase the bias but reduce the variance of the estimate resulting in a net reduction in estimation error. Adding a term that quadratically penalizes the difference between each two neighboring terms of $w$ we obtain the equation $w=\left\langle x_{t} x_{t}^{T}+\lambda M\right\rangle^{-1}\left\langle x_{t} y_{t}\right\rangle$.

Where 


$$
M=\left(\begin{array}{rrrrrrr}
1 & -1 & & & & & \\
-1 & 2 & -1 & & & \\
& -1 & 2 & -1 & & \\
& & \ldots & \ldots & \ldots & \\
& & & -1 & 2 & -1 \\
& & & & -1 & 1
\end{array}\right)
$$

An empirically determined value of $\lambda=4.4 \times 10^{-3}$ results in reduced estimation error without penalizing the heights of actual components.

\section{Appendix B: Extension to Higher Orders}

The VESPA analysis can very easily be extended to higher orders. For example, one can expand the VESPA estimation to a quadratic model of how the EEG depends on the input stimulus by replacing (A1) with a vector of $n+n(n+1) / 2$ elements, where $n$ is the window size, containing the $n$ first-order elements as before, and the $n(n+1) / 2$ second-order elements (all products of the form $x\left(t-t_{0}-i\right) x\left(t-t_{0}-j\right)$, where $0 \leq i \leq j \leq n)$. The quadratic VESPA $w$ of this same dimensionality can be solved using

$$
w=\left\langle x_{t} x_{t}^{T}+\delta I\right\rangle^{-1}\left\langle x_{t} y_{t}\right\rangle
$$

where $\delta$ is a different regularization parameter and $I$ is the identity matrix. Using values of 20 and $100 \mathrm{~ms}$ for $t_{0}$ and $n$ respectively with an empirically determined $\delta=5 \times 10^{-6}$ gives a good reduction in the estimation error. Incorporating higher than second-order terms proceeds in exactly the same way, albeit with ever larger increments in required computation time.

\section{References}

Alexander KR, Rajagopalan AS, Seiple W, Zemon VM, Fishman GA. 2005. Contrast response properties of magnocellular and parvocellular pathways in retinitis pigmentosa assessed by the visual evoked potential. Investig Ophthalmol Visual Sci 46:2967-73.

Alexander KR, Barnes CS, Fishman GA, Pokorny J, Smith VC. 2004. Contrast sensitivity deficits in inferred magnocellular and parvocellular pathways in retinitis pigmentosa. Investig Ophthalmol Visual Sci 45:4510-19.

Baseler HA, Sutter EE. 1997. M and P Components of the VEP and their visual field distribution. Vision Res 37:675-90.

Butler PD, Martinez A, Foxe JJ, Kim D, Zemon V, Silipo G, Mahoney J, Shpaner M, Jalbrzikowski M, Javitt DC. 2007. Subcortical visual dysfunction in schizophrenia drives secondary cortical impairments. Brain 130:417-30. 
Butler PD, Schechter I, Zemon V, Schwartz SG, Greenstein VC, Gordon J, Schroeder CE, Javitt DC. 2001. Dysfunction of early-stage visual processing in schizophrenia. Am J Psychiat 158: 1126-33.

Butler PD, Zemon V, Schechter I, Saperstein AM, Hoptman MJ, Lim KO, Revheim N, Silipo G, Javitt DC. 2005. Early-stage visual processing and cortical amplification deficits in schizophrenia. Archiv Gen Psychiat 62:495-504.

Chase C, Stein J. 2003. Visual magnocellular deficits in dyslexia. Brain 126:E2.

Coppola R. 1979. A system transfer function for visual evoked potentials. In: Lehmann D, Callaway E, eds. Human Evoked Potentials: Applications and Problems, NATO III: Human Factors, vol. 9. New York: Plenum Press, 69-82.

Davis AR, Sloper JJ, Neveu MM, Hogg CR, Morgan MJ, Holder GE. 2006. Differential changes of magnocellular and parvocellular visual function in early- and late-onset strabismic amblyopia. Investig Ophthalmol Visual Sci 47:4836-41.

DeAngelis GC, Ohzawa I, Freeman RD. 1993. The spatiotemporal organization of simple cell receptive fields in the cat's striate cortex. II. Linearity of temporal and spatial summation. J Neurophysiol 69:1118-35.

deBoer E, Kuyper P. 1968. Triggered correlation. IEEE Trans Biomed Eng 15:169-79.

Doniger GM, Foxe JJ, Schroeder CE, Murray MM, Higgins BA, Javitt DC. 2001. Visual perceptual learning in human object recognition areas: a repetition priming study using high-density electrical mapping. NeuroImage 13:305-13.

Doniger GM, Foxe JJ, Murray MM, Higgins BA, Javitt DC. 2002. Impaired visual object recognition and dorsal/ventral stream interaction in schizophrenia. Archiv Gen Psychiat 59:1011-20.

Foxe JJ, Simpson GV. 2002. Flow of activation from v1 to frontal cortex in humans: a framework for defining "early" visual processing. Exp Brain Res 142:139-50.

Foxe JJ, Doniger GM, Javitt DC. 2001. Early visual processing deficits in schizophrenia: impaired P1 generation revealed by high-density electrical mapping. Neuroreport 12:3815-20.

Foxe JJ, Murray MM, Javitt DC. 2005. Filling-in in schizophrenia: a high density electrical mapping and source-analysis investigation of illusory contour processing. Cerebr Cortex 15:1914-27.

Foxe JJ., Schroeder CE. 2005. The case for feedforward multisensory convergence during early cortical processing. Neuroreport 16: 419-23.

Gomez-Gonzalez CM, Clark VP, Fan S, Luck SJ, Hillyard SA. 1994. Sources of attention-sensitive visual event-related potentials. Brain Topogr 7:41-51.

Haenschel C, Bittner RA, Haertling F, Rotarska-Jagiela A, Maurer K, Singer W, Linden DEJ. 2007. Impaired early-stage visual processing contributes to working memory dysfunction in adolescents 
with schizophrenia: a study with event-related potentials and functional magnetic resonance imaging. Archiv Gen Psychiat 64:1229-40.

Hung G, Stark L. 1977. The kernel identification method: review of theory, calculation, application, and interpretation. Math Biosci 37:135-90.

Jones JP, Palmer LA. 1987. The two-dimensional spatial structure of simple receptive fields in cat striate cortex. J Neurophysiol 58:1187-1211.

Kaplan E, Lee BB, Shapley RM. 1990. New views of primate retinal function. In: Osborne N, Chader G, eds. Progress in Retinal Research, vol. 9. Oxford: Pergamon Press, 273-336.

Kaplan E, Benardete E. 2001. The dynamics of primate retinal ganglion cells. Progr Brain Res 134:17-34

Kim D, Zemon V, Saperstein A, Butler PD, Javitt, DC. 2005. Dysfunction of early-stage visual processing in schizophrenia: harmonic analysis. Schizophr Res 76:55-65.

Lalor EC, Foxe JJ. 2008. Visual evoked spread spectrum analysis (VESPA) responses to stimuli biased towards magnocellular and parvocellular pathways. Vision Res. doi:10.1016/ j.visres.2008.09.032.

Lalor EC, Kelly SP, Pearlmutter BA, Reilly RB, Foxe JJ. 2007. Isolating endogenous visuo-spatial attentional effects using the novel visual evoked spread spectrum analysis (VESPA) technique. Eur J Neurosci 26:3536-42.

Lalor EC, Pearlmutter BA, Reilly RB, McDarby G, Foxe JJ. 2006. The VESPA: a method for the rapid estimation of a visual evoked potential. NeuroImage 32:1549-61.

Lalor EC, Yeap S, Reilly RB, Pearlmutter BA, Foxe JJ. 2008. Dissecting the cellular contributions to early visual sensory processing deficits in schizophrenia using the VESPA evoked response. Schizophr Res 98:256-64.

Lee BB, Pokorny J, Smith VC, Martin PR, Valberg A. 1990. Luminance and chromatic modulation sensitivity of macaque ganglion cells and human observers. J Opt Soc Am A7:2223-36.

Lee YW, Schetzen M. 1965. Measurement of the Wiener kernels of a nonlinear system by crosscorrelation. Int J Control 2:237-54.

Marmarelis VZ. 2004. Nonlinear Dynamic Modeling of Physiological Systems. Piscataway, NJ: IEEE Press.

Marmarelis PZ, Marmarelis VZ. 1978. Analysis of Physiological Systems: The White Noise Approach. New York: Plenum Press.

Marmarelis VZ, McCann GD. 1977. A family of quasi-white random signals and its optimal use in biological system identification. Part II: Application to the photoreceptor of calliphora erythrocephala. Biol Cybern 27:57-62.

Marmarelis PZ, Naka KI. 1972. White-noise analysis of a neuron chain: An application of the Wiener theory. Science 175:1276-78. 
McKendrick AM, Sampson GP, Walland MJ, Badcock DR. 2007. Contrast sensitivity changes due to glaucoma and normal aging: low-spatial-frequency losses in both magnocellular and parvocellular pathways. Investig Ophthalmol Visual Sci 48:2115-22.

Meister M, Pine J, Baylor DA. 1994. Multi-neuronal signals from the retina: acquisition and analysis. J Neurosci Meth 51:95-106.

Murray MM, Foxe DM, Javitt DC, Foxe JJ. 2004. Setting boundaries: brain dynamics of modal and amodal illusory shape completion in humans. J Neurosci 24:6898-6903.

Sakai HM, Naka K. 1987. Signal transmission in the catfish retina. V. Sensitivity and circuit. J Neurophysiol 58: 1329-50.

Schetzen M. 1981. Nonlinear system modeling based on the Wiener theory. Proc IEEE 69:1557-73.

Schechter I, Butler PD, Zemon VM, Revheim N, Saperstein AM, Jalbrzikowski M, Pasternak R, Silipo G, Javitt DC. 2005. Impairments in generation of early-stage transient visual evoked potentials to magno- and parvocellular-selective stimuli in schizophrenia. Clin Neurophysiol 116:2204-15.

Spencer KM, Nestor PG, Niznikiewicz MA, Salisbury DF, Shenton ME, McCarley RW. 2003. Abnormal neural synchrony in schizophrenia. J Neurosci 23:7407-11.

Wiener N. 1958. Nonlinear Problems in Random Theory. Cambridge: MIT Press. 
